\title{
"I am struggling to cope with my child's behaviour and I think he may have ADHD": A brief overview of things to consider before going down the route of specialist services
}

Received: October 12, 2015; Accepted: November 10, 2015; Published: November 20, 2015

\section{Introduction}

The diagnosis of attention deficit hyperactivity disorder (ADHD) is based on the presence of a sufficient number of symptoms of unusually high levels of impulsivity, hyperactivity and inattention, that have been present since early childhood and are felt to be out of keeping with the person's developmental age and that occur in more than one setting (for example at home and in school). All children, to some extent, show behaviours of inattention, hyperactivity, and impulsivity at various times and these behaviours are known to be influenced by context.

A diagnosis rests on clinical judgement using standardised criteria, sometimes with the assistance of parental and teacher questionnaires. Increasing rates of diagnosis, rising use of stimulants, lack of evidence for long-term efficacy for stimulants, pharmaceutical marketing practices, and lack of evidence for an identifiable underlying neurological problem, have all contributed to controversy surrounding the diagnosis [1-3].

ADHD is a term that provides a description for a set of behaviours, but does not provide an explanation - it doesn't uncover the cause of these behaviours. A diagnosis alone therefore cannot tell us what treatments might be most helpful for any individual child and their family. Furthermore - although stimulants improve behaviours in the short term - outcomes after several years' treatment finds little meaningful differences between those who did or didn't receive pharmacological treatment [4-6], pointing to the importance of non-medication based approaches.

This article does not set out to provide a review of the evidence base for the treatment of ADHD, whether pharmacological or non-pharmacological. In addition, the suggestions below should not be considered as an alternative to assessment and treatment from specialists in ADHD when indicated. Instead this article is to help non-specialist practitioners with simple nonpharmacological approaches that can be tried with young people and their families where there are less severe and/or less clearcut presentations, prior to considering a referral to a specialist. This is compatible with influential UK National Institute of health and Care Excellence guidelines for ADHD treatment [7], which recommends non-pharmacological interventions as the first line treatment for mild or moderate ADHD.

\section{Sami Timimi \\ Consultant child and Adolescent \\ Psychiatrist and Lincolnshire Partnership Foundation NHS Trust}

Corresponding author:

Sami Timimi

stimimi@talk21.com

Professor, Faculty of Life, Health, and Social Sciences, University of Lincoln.

\section{Tel: 1529488061}

Citation: Timimi S. "I am struggling to cope with my child's behaviour and I think he may have ADHD": A brief overview of things to consider before going down the route of specialist services. J Child Dev Disord. 2015, $1: 1$.

\section{Possible causes of ADHD type behaviours}

Any problem that causes bodily discomfort in the child can result in behavioural problems. Thus it is helpful to ask a general practitioner do a basic screen for signs and symptoms of conditions such as anaemia, glue ear, asthma, and constipation (including investigations such as a basic blood screen, peek flow, and otoscopy). Any issue that causes psychological discomfort can also result in behavioural problems; therefore it is worth enquiring about life events, parental well-being, school issues (such as bullying and/or learning difficulties) and peer group problems.

Once more obvious causes such as the above are ruled out, it is worth trying some simple strategies that may help to make a positive difference to the child and their family, and that can be considered, where appropriate, before making a referral for specialist ADHD assessment and treatment.

\section{Simple things to try}

\section{Diet and nutrition}

Modern children's diets are high in sugars, fats, and salt and low in fibre, vitamins, minerals, and essential fatty acids. There are three components to improving young peoples' nutrition that 
have evidence to support them improving their mental health, learning and behavior $[8,9]$.

- Eliminate potential irritants - try removing all artificial additives and consider problems like lactose intolerance.

- Add any missing nutrients - try a daily multi-vitamin and mineral supplement and EPA rich Omega-3 essential fatty acids.

- Balance the diet - reduce sugars and saturated fats, increase complex carbohydrates (which help regulate blood sugar levels) and fibre (through raw fruit and vegetables), and have three balanced meals a day (breakfast is particularly important).

\section{Fresh air and exercise}

Emphasise the importance of plenty of exercise for children, particularly outdoors in green spaces, including chances for unstructured, unsupervised and 'rough and tumble' play.

\section{The importance of strong, secure relationships}

Parents need to work really hard to build positive relationships with their children, particularly when there are behavioural problems. Relationships can get stressed by high levels of negativity between a parent and child, resulting in on-going negative reinforcement, which further damages the quality of the relationship. By making a strenuous effort to notice, comment on, interact and praise their child as often as they can, children can build better self-esteem. Advise parents to have clear and immediate consequences for unwanted behaviour and work hard to stick to these and not give in - stay firm and keep calm. Advise them to avoid getting drawn into arguments, which usually adds fuel to the fire, making the situation worse. In addition, when children experience difficulty, confusion, unhappiness, excessive disobedience etc., then whatever the cause, they need the security that comes from predictability and routines.

\section{Regular positive family time}

To keep some positive family experience going, parents should make opportunities for their family to enjoy each other's company, such as going out together as a family once every weekend and having at least one meal a day together.

\section{Communication and understanding}

Encourage family members to talk to each other, but more importantly listen to each other - they are not the same thing. Encourage parents to solicit and make an effort to understand their child's point of view and help the child understand the parents. Be alert to broader family or parental unhappiness or conflict. If there are unresolved difficulties between the parents - whether they are together or separated - then these will often show themselves through the emotional state and behaviour of their children. Here, inconsistency between parents can become an obstacle to progress. You can arrange to meet with both parents and not just the one who brought the child. Try to gather the honest views of both parents (without their child being present) and remind them of the importance of both parents making decisions together and communicating these to their children.

\section{Conclusions}

Much of the literature on ADHD and its treatment, stresses biological aspects and the use of particular treatment guidelines. In this brief piece I have tried to give a shorthand guide to nonspecialist practitioners of things to consider and try with young people who have non-severe and/or less clear cut presentations (as far as diagnosis is concerned), before requesting a specialist assessment or treatment for ADHD. 


\section{References}

1 McClure I (2013) Prescribing methylphenidate for moderate ADHD. Brit Med J 347, f6216.

2 Timimi S. A Straight Talking Introduction to Children's Mental Health Problems. Ross-on-Wye: PCCS Books, 2009.

3 Timimi S, Leo J. (eds.) Rethinking ADHD: From Brain to Culture. Basingstoke: Palgrave MacMillan, 2009.

4 Government of Western Australia, Department of Health. (2010) RAINE ADHD Study: Long-term Outcomes Associated with Stimulant Medication in the Treatment of ADHD in Children. Perth: Department of Health.

5 King S, Griffin S, Hodges Z, et al. (2006) A systematic review and economic model of the effectiveness and cost-effectiveness of methylphenidate, dexamfetamine and atomoxetine for the treatment of attention deficit hyperactivity disorder in children and adolescents. Health Technol Assess 10: iii-iv, xiii-146.

6 Jensen PS, Arnold LE, Swanson JM, et al. (2007) 3-year follow-up of the NIMH MTA study. J Am Acad Child Adolesc Psychiatry 46: 9891002.

7 National Institute for Health and Care Excellence (NICE). (2008) Attention deficit hyperactivity disorder. Clinical Guideline 72. London: NICE.

8 McCann D, Barrett A, Cooper A, et al. (2007) Food additives and hyperactive behaviour in 3-year-old and 8/9-year-old children in the community: a randomised, double-blinded, placebo controlled trial. Lancet 370: 1560-1567

9 Sandford N. ADHD Without Drugs - A Guide to the Natural Care of Children with ADHD By One of America's Leading Integrative Pediatricians, 2010. 